\title{
A formação de preceptores em saúde: desenvolvendo competências interdisciplinares a partir da interprofissionalidade
}

\author{
The formation of health preceptors: developing \\ interdisciplinary competences from interprofessionality
}

\author{
Rebeca de Paula Garcia', Ana Mackartney Souza Marinho', \\ Paloma Graciano de Carvalho Moura², Jorge Luiz Barbosa Moura², \\ Paulo Marcondes Carvalho Junior ${ }^{3}$, Neilton Araujo Oliveira ${ }^{4}$ \\ ' Universidade Federal do Tocantins, Palmas (TO), Brasil. \\ 2Universidade Federal do Tocantins, Associação Brasileira de Educação Médica, Palmas (TO), Brasil. \\ ${ }^{3}$ Departamento de Ciências da Saúde, Universidade Federal de Santa Catarina, Florianópolis (SC), Brasil. \\ ${ }^{4}$ Programa de Pós-graduação em Ciências da Saúde, Universidade Federal do Tocantins, Palmas (TO), Brasil.
}

\section{RESUMO}

Durante a formação acadêmica do médico, nota-se pouco incentivo à correlação entre o conteúdo trabalhado em sala de aula e a realidade profissional, mas nos últimos anos as Instituições de Ensino Superior têm buscado integrar os diversos níveis de formação profissional, assim como as diversas especialidades e saberes, aproximando outras áreas de conhecimento e estabelecendo relações entre elas. Nessa perspectiva, oficinas e outras práticas colaborativas, com trocas de conhecimento, possibilitam interações de experiências, especialidades e habilidades que, numa construção coletiva, podem reformular modelos de atenção e de educação em saúde. O objetivo deste artigo é relatar o processo de capacitação de preceptores no "Curso de Desenvolvimento de Competência Pedagógica para a Prática da Preceptoria Médica ABEM-UFT" (CDCPPPM) que ocorreu no centro colaborador da cidade de Palmas, no estado do Tocantins. A descrição será feita a partir dos dados do perfil de cada uma das quatro turmas ao longo das quatro edições do curso. O CDCPPPM contou com a carga horária média de 180 horas cumpridas presencialmente em encontros de imersão e virtualmente através de plataformas de ensino à distância. Pautado na concepção pedagógica da problematização o curso formou noventa e um preceptores de diferentes áreas de atuação nestas quatro edições. Constatou-se que o curso, de cunho inédito na região, colaborou para o desenvolvimento de competências interdisciplinares e interprofissionais na educação em saúde, além de fomentar a continuidade e multiplicação local do processo crítico-reflexivo de formação de preceptores.

Palavras-chave: Educação em saúde. Preceptoria. Relações interprofissionais. Pesquisa interdisciplinar.

\section{ABSTRACT}

During a doctor's academic formation, there is little incentive to correlate classroom content with professional reality. However, universities have recently sought to integrate different levels of professional training, as well as various specialties and knowledge, to approach other areas of knowledge and establish relations between them. In this perspective, workshops and other collaborative practices that allow exchanges of knowledge enable interactions of experiences, specialties and skills that, in a collective construction, can reformulate models of health care and education. The objective of this article is to report on the training of preceptors in the "the ABEM-UFT Pedagogical Competency Development Course for Preceptorship Practice (PCDCPP)", which took place in the collaborating center of Palmas's city, located at Tocantins state. The description was made from the profile data of each of the four teams throughout the four editions of the course. The PCDCPP had the average hourly workload of 180 hours course classroom and virtually, through distance learning platforms. Based on the pedagogical conception of questioning, the course formed 91 preceptors from different areas of activity in these four editions. Besides fostering the continuity and local multiplication of the critical-reflexive process of teacher training, it was verified that the unprecedented regional course contributed to the development of interdisciplinary and inter-branch professional expertise in health education.

KEYWORDS: Health education. Preceptorship. Interprofessional relations. Interdisciplinary research. 


\section{Contextualização}

Durante a formação acadêmica, nota-se pouco incentivo à correlação entre o conteúdo trabalhado em sala de aula e a realidade profissional ${ }^{1}$. Contribuem para este fenômeno fatores como o processo vigente de adaptação das escolas médicas às Diretrizes Curriculares Nacionais, a unidisciplinaridade, e a falta de qualificação pedagógica dos preceptores. Presa à moldes antigos, a formação médica têm como produto profissionais incapazes de suprir as necessidades de saúde da sociedade no contexto do SUS.

As reformas curriculares nos cursos voltados à formação de profissionais de saúde consistem num dos maiores desafios relacionados à formação de recursos humanos na área. Para garantir uma formação profissional correspondente às necessidades de saúde da população, é necessária uma formação generalista, humanista, crítica e reflexiva condizente com o Sistema Único de Saúde (SUS).

Sabe-se que em um contexto de unidisciplinaridade, a atenção à saúde integral do indivíduo é prejudicada, uma vez que esse modelo reforça a relação tradicional entre membros de equipe, desvalorizando a colaboração do outro.

A integração entre disciplinas, saberes e práticas consiste num desafio presente no cotidiano dos profissionais da saúde, ao desenvolverem o seu trabalho em equipe. Garcia et al. ${ }^{2}$ (p. 147-155) diz:

[...] na interdisciplinaridade as ações seriam planejadas em função das necessidades do grupo populacional a ser atendido e não se limitaria às definições apriorísticas de papéis de cada profissional. De modo esquemático, o que diferenciaria este conceito do de transdisciplinaridade seria a permanência da dominância de determinados saberes.

As práticas colaborativas permitem interações onde conhecimentos, especialidades, experiências e habilidades, são compartilhados numa lógica baseada em valores humanistas. Estes buscam tornar a pessoa o centro da atenção profissional. Nesse sentido o trabalho interprofissional é uma resposta possível para esses impasses, reagrupando conhecimentos que estão dispersos, evitando também a duplicação de cuidados ${ }^{3}$.

Nesta perspectiva, Ceccim e Feuerwerker ${ }^{4}$ consideram que as Instituições de Ensino Superior têm buscado integrar os diversos níveis de formação profissional, assim como as diversas especialidades e saberes, aproximando outras áreas de conhecimento ao estabelecer relações horizontais. Para isso, é fundamental a humildade e a disponibilidade num movimento para reconhecer dificuldades entre as partes, de posições diferentes entre os atores e nas interações entre estes ${ }^{2}$.

A construção coletiva é fundamental para a reconsideração dos modelos de atenção e de educação em saúde, através da interdisciplinaridade nas práticas profissionais crítico-reflexivas sobre o trabalho em saúde, permitindo que o cotidiano de relações da organização da gestão setorial e estruturação de cuidados à saúde, se incorpore ao aprender e ao ensinar, formando profissionais não só para a área da saúde como também para o SUS4.

Por este motivo, Anastasiou e Alves ${ }^{1}$ falam da intensificação da realização de oficinas pedagógicas que permitam a troca de conhecimentos envolvendo um conjunto de pessoas, na construção de saberes integrados, baseados em objetivos comuns. De acordo com Garcia et al. ${ }^{2}$, o desenvolvimento de competências e habilidades apontadas pelas Diretrizes Curriculares Nacionais para o Curso de Medicina poderia ser efetivado, através da integralidade, modificando a produção de saberes e da formação profissional, onde deve ser claro que uma única ciência não é capaz de abranger as necessidades de uma pessoa ou da sociedade.

Por este ângulo, a formação pedagógica do preceptor é fundamental para a efetivação do SUS, pois através dos estágios realizados nestes cenários de prática o discente tem oportunidade de discutir e elaborar estratégias para a superação de problemas de saúde da população. A visão da personalidade integral do indivíduo é desenvolvida conforme o rompimento da dissociação entre teoria e prática. 
Deste modo, ressalta-se a necessidade de estudos voltados à preceptoria em saúde, assim como o desenvolvimento de suas competências pedagógicas, uma vez que a mudança na formação, conforme Ceccim e Feuerwerker ${ }^{4}$, está pautada na tríade ensino-serviço-comunidade. A integralidade do cuidado no SUS é capaz de reestruturar os estabelecimentos e as organizações do setor da saúde de modo articulado, interprofissionalmente, honrando os dispositivos e princípios do mesmo.

Anastasiou e Alves ${ }^{1}$ ressaltam a necessidade da conversa, capaz de levar à superação das dificuldades encontradas ao longo do processo. Além disso, a possibilidade de diálogo revela a necessidade de estruturação de equipes de saúde garantindo trocas e inter-relações profissionais, num processo de tensão e vinculação da interdisciplinaridade e da interprofissionalidade no SUS.

Na promoção da interdisciplinaridade, o preceptor atua diretamente na formação do aluno, apesar da falta de capacitação para o exercício desta função. Costa et al. ${ }^{5}$ afirma que existe uma deficiência no domínio da área educacional no desempenho do docente de Medicina. A atuação docente na área médica se restringe à reprodução de modelos considerados válidos aprendidos anteriormente e à experiência prática cotidiana. E ainda diz que é necessário planejar uma formação pedagógica que integre experiências e vivências docentes com a discussão e o debate de princípios teóricos de educação.

Dentro desta perspectiva, para o grande estudioso da educação, Perrenoud ${ }^{6}$, o principal recurso do professor é a "postura reflexiva", definida como sua capacidade de observar, de regular, de inovar, de aprender com os outros e com a experiência. Para isso são necessários projetos que criem estratégias de discussão e troca de experiência, que promovam o desenvolvimento de habilidades pedagógicas e que possam ser inseridos na já disputada carga horária dos preceptores das escolas médicas brasileiras.

Chazan et al.7 (p. 102) ainda diz: "Os docentes precisam se aventurar a passar por processos de ensino aprendizagem que reconfigurem seus "velhos habitus", considerando que, para formar novos médicos, ser um deles é necessário e importante, mas certamente já não é suficiente".

Há a necessidade de mudar a prática médica através da valorização do processo de ensino-aprendizagem dos cenários de prática, capacitando docentes e preceptores para a formação profissional crítica e voltada à saúde.

Vale ressaltar que este relato de experiência tem como objetivo descrever a organização e construção do Curso de Desenvolvimento de Competência Pedagógica para a Prática da Preceptoria Médica (CDCPPPM) no Tocantins, nos anos de 2012 a 2015, com ênfase na interdisciplinaridade e interprofissionalidade dos preceptores (discentes) e equipe (docentes) do Curso.

Durante a gestão de 2010-2012 a associação Brasileira de Educação Médica (ABEM) buscou dar continuidade à Carta de Acordo BR \LOA \1121.001, com o Projeto Preceptoria. Em sua primeira fase, durante os anos de 2010 a 2012 o Projeto objetivou realizar um programa para o desenvolvimento de competências pedagógicas para a prática da preceptoria na Residência Médica pautado pelos princípios do SUS e competências gerais das Diretrizes Curriculares Nacionais de graduação de medicina ${ }^{8}$.

Nesta fase, foi constituído um Núcleo Docente Estruturante (NDE) com 44 membros responsáveis pela implantação do Curso (CDCPPPM) em 12 centros colaboradores (CC), que participaram da organização e oferta das primeiras turmas do curso em locais estratégicos nacionais. A Universidade Federal do Tocantins foi um destes centros colaboradores.

\section{Descrição da experiência}

Este relato de experiência é fruto da participação e observação dos autores no "Curso de Desenvolvimento de Competência Pedagógica para a Prática da Preceptoria" da ABEM em parceria com a Universidade Federal do Tocantins (UFT). 
A partir dos acervos do Curso, foram colhidos dados dos perfis de cada turma no período de 2012 a 2015, totalizando quatro turmas. A carga horária do curso está composta por 180 horas, sendo que 60 horas são presenciais com encontros de imersão, podendo ser três encontros de dois dias consecutivos com intervalo de seis semanas, ou dois encontros de três dias consecutivos com intervalos de oito semanas. As demais 120 horas, à distância, são realizadas através de ambientes virtuais de aprendizagem (AVA) operacionados pela plataforma moodle. A equipe organizadora e executante do curso, contava com as seguintes figuras:

COORDENADORES: têm a atribuição de coordenar as ações que permitam o desenvolvimento integral do Projeto, elaborar relatórios, divulgar os resultados e prestar contas.

SUPERVISORES: têm a atribuição de acompanhar e avaliar o desenvolvimento integral dos cursos, presencial e à distância, busca o alinhamento das práticas pedagógicas nos diferentes Centros e interage com a Coordenação do Projeto com vistas ao alcance dos objetivos.

SUPERVISOR DE EAD: acompanha, assessora e supervisiona o desenvolvimento das atividades no Ambiente Virtual do curso.

ORIENTADORES DE APRENDIZAGEM (OAs): acompanha, apoia e orienta o ambiente presencial e à distância as atividades dos tutores, é o responsável pelo feedback formativo ao tutor.

ASSESSOR PEDAGÓGICO: tem a atribuição específica de auxiliar no acompanhamento e análise de indicadores de resultado do projeto.

FACILITADORES DE EAD - atuam no suporte às atividades da equipe do NDE, operacionalizam o planejamento do curso no ambiente virtual, monitoram os fóruns à distância e organizam os materiais produzidos.

TUTORES - Após capacitação, desenvolvem o curso para preceptoria nos Centros Colaboradores e de Referência e auxiliam na sistematização dos relatórios finais e consolidação dos dados.

TUTORES GESTORES: Tutores com a responsabilidade pela organização logística do Curso nos Centros de Referência além de apoiar os Tutores Junior no desenvolvimento das atividades presenciais e à distância.

O CDCPPP é estruturado em três eixos: cuidado, educação e gestão do trabalho em saúde. Sua concepção pedagógica norteadora é a da problematização, baseando o ensino e a aprendizagem nos problemas extraídos do contexto da prática. As estratégias utilizadas são as metodologias ativas, com diferentes recursos didáticos, como: situação problema, construção de relatos de prática, exposição dialogada, dinâmicas de grupo, dramatizações, recursos audio-visuais, dentre outros. Tanto nos momentos presenciais quanto à distância, as experiências de ensino e aprendizagem estão organizadas em diferentes grupos de trabalho, favorecendo a integração e o desenvolvimento de capacidades colaborativas para o trabalho em equipe. Há também momentos de atividades individuais, auto-dirigidas e de busca ativa do conhecimento. Como proposta de trabalho final do Curso, cada preceptor elabora um projeto de intervenção, com objetivo de qualificação da formação em seu cenário de atuação. Essa é uma das avaliações do Curso, porém a avaliação não é pontual, e sim contínua, sendo um componente importante desse processo formativo, permitindo o aprimoramento das estratégias de ensino adotadas, além de uma auditoria do próprio desempenho dos educandos ${ }^{9}$.

\section{Resultados e impactos}

$1^{\text {a }}$ Turma (2012):

A $1^{\text {a }}$ turma do estado do Tocantins era formada por preceptores da Residência Médica da UFT nas seguintes especialidades: Pediatria (10), Cirurgia (6), Clínica Médica (5), Ginecologia-Obstetrícia (2) e Programa de Saúde da Família (2), além de 
duas enfermeiras convidadas da ETSUS (Escola Tocantinense do SUS), que auxiliariam na estruturação da continuidade local do Curso, favorecendo mais essa parceria, e também a ampliação do público-alvo local para o multiprofissional, no futuro.

A Equipe (Núcleo Docente Estruturante do Tocantins: NDE-TO) neste ano foi composta por dois tutores (sendo a tutora local a primeira autora deste estudo, e o outro tutor externo), um orientador de aprendizagem (OA, responsável por acompanhar, apoiar e orientar os tutores) e uma supervisora, que era também a coordenadora nacional do Projeto Preceptoria ABEM nacionalmente. Todos esses médicos, porém de especialidades diferentes.

$2^{\text {a }}$ Turma (2013):

Nesse ano, o estado do Tocantins foi pioneiro na continuidade do Projeto Preceptoria ABEM com parcerias locais (ETSUS, além do já Centro Colaborador UFT), e para isso fez algumas adaptações à realidade local. O público-alvo estendeu-se de preceptores da Residência Médica, também para os de Internato e Graduação, ajustando-se então o nome para "Curso de Desenvolvimento de Competência Pedagógica para a prática da Preceptoria Médica".

A turma era formada por preceptores das seguintes especialidades: Pediatria (7), Cirurgia(7), Clínica Médica(7), Ginecologia-Obstetrícia(3), Programa da Saúde da Família (4), Patologia (1) e Ortopedia (1), além de um convidado do Internato Rural da UFT, odontólogo, e duas enfermeiras convidadas da ETSUS, que auxiliaram na estruturação da continuidade local do Curso e também no planejamento da ampliação do público-alvo para o multiprofissional, para o ano seguinte.

A Equipe (NDE-TO) neste ano foi composta pelo dobro de pessoas (oito): quatro novos integrantes, como tutores, e os quatro já existentes. Os novos tutores foram preceptores formados na turma anterior, sendo uma deles específica para o AVA. Dos integrantes prévios, dois mudaram de função: a primeira autora deste estudo, tornou-se a coordenadora local, o outro tutor (do ano anterior) tornou-se também um orientador de aprendizagem (ficando, então, dois OAs) enquanto à supervisão, não houve mudança em relação ao ano anterior. Dos oito, eram sete médicos, de diferentes especialidades, e uma enfermeira. Avanços como a parceiria do ETSUS e a utilização de Ambiente Virtual de Aprendizagem próprio o Telessaúde - UFT, e não mais a plataforma Telessaúde da UERJ, foram passos importantes para tornar-se um centro de referência.

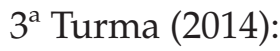

Nesse ano, o estado do Tocantins deu continuidade ao Projeto Preceptoria ABEM com parcerias locais (ETSUS, além do já Centro Colaborador UFT), e fez mais algumas adaptações à realidade local. O público-alvo estendeu-se de preceptores da Residência Médica também para Multiprofissional, além dos de Internato e Graduação, incluindo agora os Cursos de Enfermagem e Nutrição da UFT, ajustando-se então o nome para "Curso de Desenvolvimento de Competência Pedagógica para a prática da Preceptoria em Saúde".

A turma era formada por preceptores das seguintes áreas: Pediatria (6), Cirurgia (4), Clínica Médica (2), Ginecologia-Obstetrícia (1), Programa de Saúde da Família (2), Anestesia (2), Ortopedia (1), Enfermagem (2), Nutrição (1), Fisioterapia (1), Psicologia (1) e Odontologia $\left({ }^{1}\right.$, além de uma cirurgiã dentista convidada do Curso de Especialização FAIMER Brasil ${ }^{\circledR}$, que auxiliaria na estruturação de um curso semelhante, para o público-alvo multiprofissional, em sua região (Nordeste).

$4^{\text {a }}$ Turma (2015):

Nesse ano, houve a continuidade ao Projeto Preceptoria ABEM com a sua fase II, nacionalmente. A UFT, juntamente com os demais Centros Colaboradores da fase I, se tornaram Centros de Referência; e mais novos sete Centros se incorporaram como Colaboradores. O público-alvo no Tocantins manteve-se de preceptores da Residência 


\section{ijhe}

Médica e Multiprofissional, além dos de Internato e Graduação, incluindo os Cursos de Enfermagem e Nutrição da UFT, mantendo-se o nome "Curso de Desenvolvimento de Competência Pedagógica para a Prática da Preceptoria em Saúde".

A turma era formada por preceptores das seguintes áreas: Pediatria (4), Cirurgia (3), Clínica Médica (7), Ginecologia-Obstetrícia (2), Programa da Saúde da Família (2), Anestesia (2), Psiquiatria (1), Dermatologia (1), Enfermagem (3), Nutrição (2) e Biologia (1).

A Equipe (NDE-TO) neste ano foi composta por quatro novos tutores, sendo dois "juniores" (um fisioterapeuta e uma psicóloga) e dois "observadores" (ambos médicos), todos formados na turma anterior; dos três tutores "sêniores" de 2014, continuaram dois; a primeira autora deste estudo se tornou a orientadora de aprendizagem e o OA do ano anterior se tornou o supervisor.

\section{Considerações finais}

Conclui-se, com este relato de experiência, que Cursos de Formação de Preceptores em um modelo educacional crítico-reflexivo e com abordagem holística conduzida por profissionais da saúde e educação, desde a formação de seus tutores, colabora para o processo de desenvolvimento de competências interdisciplinares e interprofissionais na educação em saúde.

No Tocantins, o CDCPPPM foi pioneiro nesses moldes. Integrou diferentes áreas de atuação e ao longo das quatro edições formou preceptores com habilidades pedagógicas voltadas ao ensino médico. Com a continuidade do curso, o objetivo é aprofundar e analisar outros apectos, a fim de abordar de forma qualitativa e quantitativa, mais dados do Projeto.

\section{Referências}

1. Anastasiou LGC, Alves LP. Estratégias de ensinagem. In: Anastasiou LGC, Alves LP. Processos de ensinagem na universidade. Joinville: Univille; 2007. p. 73-107.

2. Garcia MAA, Pinto ATBCS, Odoni APC, et al. A interdisciplinaridade necessária à educação médica. Rev Bras Educ Med. 2007;31(2):147-55. http://dx.doi.org/10.1590/S0100-55022007000200005.

3. Furtado JP. Arranjos institucionais e gestão da clinica: princípios da interdisciplinaridade e interprofissionalidade. J Ment Health. 2011;1(1):178-89.

4. Ceccim RB, Feuerwerker LCM. O quadrilátero da formação para a área da saúde: ensino, gestão, atenção e controle social. PHYSIS: Rev. Saúde Coletiva. 2004;14(1):41-65. http://dx.doi.org/10.1590/S0103-73312004000100004.

5. Costa NMSC, Cardoso CGLV, Costa DC. Concepções sobre o bom professor de medicina. Rev Bras Educ Med. 2012;36(4):499505. http://dx.doi.org/10.1590/S0100-55022012000600008.

6. Perrenoud P. A prática reflexiva no ofício do professor: profissionalização e razão pedagógicas. Porto Alegre: Artmed; 2002.

7. Chazan ACS. As dores e delícias da formação médica: um estudo de caso sobre a qualidade de vida dos estudantes de Medicina da Faculdade de Ciências Médicas da UERJ [tese]. Rio de Janeiro: Escola Nacional de Saúde Pública Sergio Arouca; 2015.

8. Associação Brasileira de Educação Médica. Caderno de ensinagem do Tutor. Rio de Janeiro; 2016.

9. Afonso DH, Silveira LM. Projeto Desenvolvimento de Competência Pedagógica para a Prática da Preceptoria: Fase II. Protocolado em 30/10/2012 sob nº 25000.192026/2012-48. - Rio de Janeiro: ABEM; 2014. Carta Acordo assinada em $15 / 05 / 14$.

10. Paula RG. Avaliação do desenvolvimento pedagógico dos preceptores de um curso de preceptoria em saúde no Estado do Tocantins [dissertação]. Tocantins: Universidade Federal do Tocantins; 2016. p. 15-50 [citado em 2016 Jun 1]. Disponível em: http://repositorio.uft.edu.br/handle/11612/212

\section{Contribuição dos autores}

Todos os autores participaram do planejamento e execução do trabalho, leram e aprovam a versão final submetida ao Interdisciplinary Journal of Health Education (IJHE). 